\title{
Perfectionism and Psychological Distress: A Modeling Approach to Understanding their Therapeutic Relationship
}

\author{
Chantal A. Arpin-Cribbie · Jane Irvine $\cdot$ Paul Ritvo \\ Robert A. Cribbie · Gordon L. Flett · Paul L. Hewitt
}

Published online: 27 February 2008

(C) Springer Science+Business Media, LLC 2007

\begin{abstract}
The present study assessed the effectiveness of a web-based psychoeducational intervention protocol for decreasing levels of perfectionism and psychological distress. Different levels of therapeutic intervention (no treatment, general stress management intervention, general stress management intervention plus cognitive behavioral intervention) were provided to perfectionistic participants over a 10 -week period. It was found via a longitudinal structural equation model that higher levels of therapeutic intervention predicted greater improvements in perfectionism and psychological distress. Further, amount of improvement in trait perfectionism and perfectionistic automatic thoughts was highly related to amount of improvement in psychological distress. The findings attest to the potential usefulness of a web-based intervention that combines a general stress management intervention with a cognitive behavioral intervention.
\end{abstract}

Keywords Perfectionism - Distress - Intervention - Structural equation model

\section{Introduction}

Currently, there exist various conceptualizations of perfectionism and how it affects general well-being. Two theories in particular have proved informative-namely,

C. A. Arpin-Cribbie $(\bowtie) \cdot$ J. Irvine $\cdot$ R. A. Cribbie $\cdot$ G. L. Flett

Department of Psychology, Behavioural Sciences Building, York University, 4700 Keele Street,

Toronto, ON, Canada M3J 1P3

e-mail: arpin@yorku.ca

P. Ritvo

School of Kinesiology and Health Science, York University, 4700 Keele Street, Toronto, ON,

Canada M3J 1P3

P. L. Hewitt

University of British Columbia, Vancouver, Canada 
the multidimensional theories of perfectionism outlined by Frost et al. (1990) and by Hewitt and Flett (1991). These particular theories have been used to obtain a greater understanding of the psychological distress of post-secondary students, in particular the high prevalence of perfectionistic tendencies in academic settings. The proposition that perfectionism is a multidimensional construct has led to significant advancements in our understanding of perfectionism and the way it affects states of psychological well being or distress. This multidimensional conceptualization of perfectionism takes account of both intrapersonal and interpersonal aspects in examining the relative cost to benefit ratio of perfectionism on an individual's level of adaptive functioning. Not surprisingly, these multidimensional measures tend to focus on the negative aspects of perfectionism (see Flett and Hewitt 2006).

\section{Multidimensional Measures of Perfectionism}

Hewitt and Flett's (1991) Multidimensional Perfectionism Scale (MPS) is one of the more widely used measures of perfectionism whose psychometric properties have been repeatedly validated. The three MPS subscales assess self-oriented perfectionism, other-oriented perfectionism, and socially prescribed perfectionism. As outlined by Hewitt and Flett, "the primary difference among these dimensions is not the behavior pattern per se, but the object to which the perfectionistic behavior is directed" (p. 457). Individuals who score high on self-oriented perfectionism tend to hold themselves to excessively high standards and are highly motivated to attain these standards. Individuals who score high on other-oriented perfectionism will tend to project their expectations for perfection onto others and hold unrealistic beliefs and expectations which they use to evaluate the performance of significant others. Lastly, individuals who score high on socially prescribed perfectionism believe that others around them have exceedingly high expectations and standards, which they use to gauge performance.

The multidimensional conceptualization of perfectionism proposed by Frost et al. (1990) has components that vary somewhat from those proposed by Hewitt and Flett (1991). Frost et al. developed a six-factor assessment measure that evaluates two primary dimensions that include perfectionism directed toward the self, and the perception on the part of an individual of parental demands directed toward the self.

Though Frost et al. (1990) clearly outline the multidimensional nature of perfectionism, one dimension is of particular interest to the proposed studynamely, the 'Concern over Mistakes' subscale. Although perfectionism is generally associated with the setting of excessively high standards of performance, the 'Concern over Mistakes' construct is most directly related to this concept and highly related to the symptoms of psychopathology. Increasing concern over mistakes may be characterized by a dichotomous thinking style whereby one's performance is viewed either as perfect or as being completely insignificant with little or no cognitive middle ground. This cognitive dimension was also addressed by Ellis (2002). Ellis suggested that perfectionism becomes dysfunctional as a result of the irrational importance attached to perfectionism. What is clearly evidenced is the need to address this cognitive dimension as part of any cognitive intervention for 
the treatment of perfectionism. More specifically, a cognitively based intervention should try to reduce the distorted importance ascribed to perfectionism.

\section{Perfectionism, Anxiety, and Depression}

Perfectionism, as one set of dysfunctional cognitions, has been linked to adjustment and achievement (Hewitt and Flett 1991). Hewitt and Flett have also tied perfectionism to what has been characterized as a pervasive neurotic style, and has been correlated with and linked to a variety of negative outcomes such as feelings of failure, guilt, or shame, as well as to more serious forms of psychopathology such as in depression, alcoholism, and personality disorders. A perfectionist's tendencies to engage in behavior or to adopt cognitive frameworks such as setting/striving for extremely high standards, critically evaluating themselves harshly, overgeneralizing failure, and adhering to all-or-none thinking has been found to be associated with adjustment difficulties. More specifically, self-oriented perfectionism has been linked to increased symptoms of anxiety (Flett et al. 1989; Hayward and Arthur 1998) and depression (Hayward and Arthur 1998; Hewitt and Dyck 1986; Hewitt and Flett 1990; Hewitt et al. 1990). In addition, socially prescribed perfectionism has consistently been linked to measures of both anxiety and depression (Flett et al. 1991, 1994, 1997; Hayward and Arthur 1998; Joiner and Schmidt 1995; Martin et al. 1996).

\section{Perfectionism Management Programs}

\section{Aim of Cognitive Behavioral Interventions}

Associations have been discovered between cognitions, personality characteristics, and other psychosocial variables on the development, the perpetuation, or both, of experiences of psychological distress. Cognitive behavioral models are based on the assumption that there are interacting processes occurring and assume that cognitions impact on one's emotions, physiological processes, behavior, and overall psychosocial well-being (Deale et al. 1997). According to a cognitive behavioral view, the way one interprets one's experiences can play an important role in one's perceived experience of personal distress. Thus, a cognitive behavioral approach views an individual's beliefs as not only the result of an underlying cause, but also as possible important etiological factors in and of themselves (Sharpe 1996).

Cognitive behavioral therapies are designed to alter both the cognitions and the behaviors of a given individual, particularly the dysfunctional cognitions and behaviors that serve to prolong and maintain one's psychosocial distress (Deale et al. 1997). Studies have shown that cognitive behavior therapy can improve symptoms in individuals whose perceptual experiences and coping behaviors inhibit their recovery (Butler et al. 1991; Deale et al. 1997; Friedberg 1996; Friedberg and Krupp 1994; Fukuda et al. 1994; Sharpe 1996). More specifically, as it relates to the present study, "treatment programs should not only strive to reduce overall levels of perfectionism, but they should also focus directly on the perfectionist's tendency to 
engage in excessive cognitive rumination about the need to attain perfection. Cognitive-behavioral interventions may be particularly useful in this regard" (Flett et al. 1998, p. 1377).

\section{Effectiveness of Interventions on Perfectionistic Tendencies and Cognitions}

Though many studies have examined the negative correlates and associated adjustment difficulties experienced by perfectionistic individuals, it is surprising how little work has been done to empirically validate treatment interventions. Of the few studies that have been done however, results tend to support the effectiveness of CBT interventions in reducing perfectionism and depression (Ferguson and Rodway 1994; Richards et al. 1993). More recently, in an empirical study conducted with university students where an eight session CBT group therapy intervention was used to modify experiences of perfectionism, statistically significant decreases in perfectionism, depression, and anxiety at post-intervention were found when compared to pre-intervention levels (Kutlesa 2002). A brief overview of the literature on interventions for perfectionistic individuals suggests CBT is the most frequently recommended form of treatment with this client population (Barrow and Moore 1983; Flett et al. 1991, 1994; Halgin and Leahy 1986).

Blatt and Zuroff (2002) explored in greater detail the role perfectionism may play in the therapeutic process. To this end, they conducted research using a dataset from the Treatment for Depression Collaborative Research Program (TDCRP) sponsored by the National Institute of Mental Health (NIMH). An analysis of the dataset indicated that perfectionism predicted less improvement on depression from baseline to post treatment (Blatt and Zuroff 2002). Blatt and Zuroff concluded that irrespective of the type of treatment (pharmacotherapy, psychotherapy, or placebo), preoccupation with introspective issues of self-definition and self-worth, as measured by the perfectionism scale of the Dysfunctional Attitudes Scale, significantly impeded response to short term treatment for depression. Additional research has also demonstrated that trait dimensions of perfectionism have been linked with persistent forms of depression (Hewitt et al. 1998). A study by Cox and Enns (2003) showed that perfectionism was still correlated with residual symptoms of depression following what seemed to be a relatively successful CBT intervention. Taken together, this research suggests that while perfectionism is associated with a poorer response to treatment, psychotherapy can lead to decreases in perfectionism and the associated negative mood states.

\section{Evidence for the Effectiveness of Self-directed Interventions}

The advent of computer-based technological advancements has offered new methods for disseminating psychological treatments. Through the use of web-based interventions, many barriers associated with more traditional face-to-face psychological treatments are surmounted, particularly those related to accessibility (in terms of location, time) and cost. Internet based interventions thus far seem to 
focus on psychobehavioral issues with outcomes that can be assessed in terms of behavioral change and/or symptom improvement (Ritterband et al. 2003). Although it appears certain that web-based interventions will likely gain momentum and significance in the treatment of psychobehavioral problems in the future, it is disconcerting to note that there has been, to date, very little research that addresses the feasibility and efficacy of these interventions.

\section{The Present Study}

The primary goal of the present study was to empirically assess the effectiveness of a web-based psycho-educational intervention protocol for decreasing levels of perfectionism and psychological distress. Specifically, it was expected that changes in perfectionism scores from pretest to posttest would be related to the level of therapeutic intervention being presented to the participants. Specifically, those receiving no treatment (NT) would improve less than those receiving a general stress management intervention (GSMI), and those receiving GSMI would improve less than those receiving the GSMI and the cognitive behavioral intervention (GSMI/CBI). This same effect was also expected to affect psychological distress (depression, anxiety, negative thoughts), with NT participants improving less than GSMI participants, and GSMI participants improving less than GSMI/CBI participants. Lastly, it was expected that changes in perfectionism from baseline to posttest would be significantly positively related to changes in psychological distress. This study tested the validity of these hypotheses using a structural model where it is possible to evaluate the hypotheses of the study simultaneously, instead of independently. Clearly, a primary advantage of structural equation modeling is that it allows the possibility of testing multiple related hypotheses simultaneously, wherein researchers (accurately) acknowledge that different behaviors, and behavioral changes, do not occur in isolation but instead are intricately connected.

\section{Methods}

\section{Participants}

Participants $(N=83)$ were distributed approximately evenly across all three conditions $(\mathrm{NT}=24, \mathrm{GSMI}=29, \mathrm{GSMI} / \mathrm{CBI}=30)$. All participants were enrolled in an undergraduate introductory psychology class at a large Canadian university. The gender breakdown for each level of therapeutic intervention was similar with approximately $30 \%$ of each being male. No significant differences were noted across the levels of therapeutic intervention on the demographic data collected. As such, the demographic data will be reported for the entire sample. Participants ranged between 18 and 48 years of age, with a mean age of $20.14(S D=4.14)$. The sample consisted primarily of individuals who identified themselves as being single $(97.4 \%)$, and who were in either first $(70.1 \%)$ or second $(20.8 \%)$ year in their university program. 
Students were asked to volunteer for participation in the study if they felt their academic or personal lives were negatively affected by perfectionism. Potential participants completed an initial screening set of measures and were informed that only some of them would be selected to take part in the actual study. No indication was provided to participants as to the actual criteria used to determine eligibility. Participants were screened using the 'Perfectionism Cognitions Inventory' (see below) and had to obtain a score greater than or equal to one standard deviation above the mean in order to be eligible for participation in the study. Participants who were not comfortable with the English language or the use of web-based programs were excluded from the study.

\section{Measures}

Multidimensional Perfectionism Scale, 'Concern over Mistakes' Subscale (CM)

The Multidimensional Perfectionism Scale (Frost et al. 1990) is a 35-item questionnaire used to assess six perfectionism dimensions. The subscale of interest in this study is the CM scale. The CM subscale demonstrated high internal consistency $(\alpha=.87)$. Participants are asked to indicate to what extent they agree or disagree with statements on a 5-point Likert scale.

\section{Multidimensional Perfectionism Scale}

The Multidimensional Perfectionism Scale (Hewitt and Flett 1991) is a 45-item questionnaire used to assess three dimensions of perfectionistic behavior. The three subscales assessed by the Multidimensional Perfectionism Scale are (a) selforiented perfectionism (SOP), (b) other-oriented perfectionism (OOP), and (c) socially prescribed perfectionism (SPP). For the purposes of this study only the SOP and SPP subscales are utilized. The $\alpha$ 's were .85 for the SOP subscale and .86 for the SPP subscale. Participants are asked to indicate to what extent they agree or disagree with statements on a 7-point Likert scale.

\section{Perfectionism Cognitions Inventory (PCI)}

The PCI (Flett et al. 1998) is a 25-item questionnaire used to assess the frequency of 'automatic perfectionistic thoughts'. This measure indirectly gauges the extent to which an individual engages in cognitive evaluations between the ideal, perfectionistic self and the current self or situation. Participants are asked to indicate how frequently a given thought has occurred in the past week on a 5-point scale. Extensive evidence attests to the psychometric properties of the PCI in student samples and clinical samples (Flett et al. 1998, Flett et al. in press). The PCI had an $\alpha$ of .86 in this study. 


\section{Automatic Thoughts Questionnaire (ATQ)}

The ATQ (Hollon and Kendall 1987) is a 30-item questionnaire used to assess automatic negative thoughts about the self by measuring the cognitive selfstatements of an individual. The ATQ examines four aspects of automatic thoughts: these are (a) personal maladjustment and desire for change, (b) negative selfconcepts and negative expectations, (c) low self-esteem, and (d) helplessness. This scale demonstrated high internal consistency $(\alpha=.96)$. Participants are asked to rate the frequency of a given thought during the previous week on a 5-point Likert scale.

\section{Center for Epidemiologic Studies-Depressed Mood Scale (CESD)}

The CESD (Radloff 1987) is a 20-item questionnaire used to assess depressive symptomatology. The internal consistency of the scale in this study was very good $(\alpha=.87)$. Participants are asked to indicate the number that best describes the way they have felt on the given items in the past week on a four-point scale.

\section{Beck Anxiety Inventory (BAI)}

The BAI (Beck et al. 1988) is a 21-item questionnaire used to assess clinical anxiety. This scale demonstrated high internal consistency with an alpha coefficient of .90. Participants are asked to rate the degree to which he or she has been bothered by every given symptom during the previous week on a four-point Likert scale.

\section{Procedure}

Following informed consent, participants completed the baseline set of questionnaires. Participants were then assigned randomly to one of three levels of therapeutic intervention: (1) GSMI/CBI; (2) GSMI; or (3) NT. It is important to note that the level of therapeutic intervention is an ordinal variable since the level increases (ordinally) across the conditions. The intervention materials were available online to the GSMI/CBI and GSMI conditions for 10 weeks. For both intervention groups the material was displayed in chapter format, with each chapter as a PDF file on the web site that could only be accessed via personal ID and password. Approximately one week following the intervention period, all participants completed the post intervention measures (identical to the pretest measures).

\section{Treatment Interventions}

The General Stress Management intervention covered two main topics that included: 'Learning Not to Stress Yourself Out', and 'Bouncing Back Better'. These main topic areas were addressed by chapters covering the following: (a) 
Recognizing and dealing with stress (recognizing how stress uniquely 'gets to' you and learning what helps you to reduce stress), (b) Dealing with distractions and distractibility (seeing how stress gets you distracted and discovering what you can do to maintain focus), (c) Changing your stressors (learning to relax... progressive relaxation and breath-focused relaxation), (d) Exercise (getting started and monitoring your progress), (e) Sleep (healthier sleep makes your brain work better), and (f) Meditation (maintaining awareness and balance).

The Cognitive Behavioural Intervention included all materials found in the General Stress Management intervention, as well as materials aimed specifically at modifying perfectionistic beliefs and their related effects on mood. The CBT intervention covered three main topics: 'Rediscovering Clear Thinking', 'Learning Not to Stress Yourself Out', and 'Bouncing Back Better'. These topic areas were addressed by the following chapters, in addition to all those already found in the general stress management intervention: (a) Living in the real world (checking out your interpretations), (b) Living in the world of shoulds (examining and reevaluating expectations \& the importance of personal choice), (c) Work out your mind (recognizing how certain ways of thinking cause anxiety and depression), (d) Dealing with negative moods (three skills for dealing with negative moods), (e) When a want becomes necessity (keeping perspective on desires), and (f) Dealing with academic and performance anxiety (helping you do and feel your best). Material that was presented in the general stress management intervention was also offered in the CBT intervention but with relevant cognitive components retained, while these were omitted from the stress management intervention to prevent from contamination across intervention type.

\section{Structural Model}

A structural model was used to understand the connection between changes in perfectionism and changes in psychological distress (see Fig. 1). More specifically, pre and post measures of SOP, SPP, CM, and PCI were used to model a latent perfectionism construct, while pre and post measures of ATQ, BAI and CESD were used to model a latent psychological distress construct. Level of therapeutic intervention was included as an ordinal variable, which is appropriate in the current model given that it is only acting as a predictor (if it was acting as an outcome variable a more appropriate estimator for discrete data would be necessary). It was hypothesized that changes in perfectionism and psychological distress would be predicted from the level of therapeutic intervention received by the participants, and that changes in perfectionism would correlate with changes in psychological distress. More specifically, those receiving higher levels of therapeutic intervention would show greater improvement in perfectionism and psychological distress, and those showing greater improvement in perfectionism would also show greater improvement in psychological distress.

Structural equation modeling was conducted with the AMOS 6 software package (Arbuckle 2005). The fit of the model was evaluated using the likelihood ratio statistic $\left(\chi^{2}\right)$, comparative fit index (CFI, Bentler 1989), incremental fit index (IFI, 


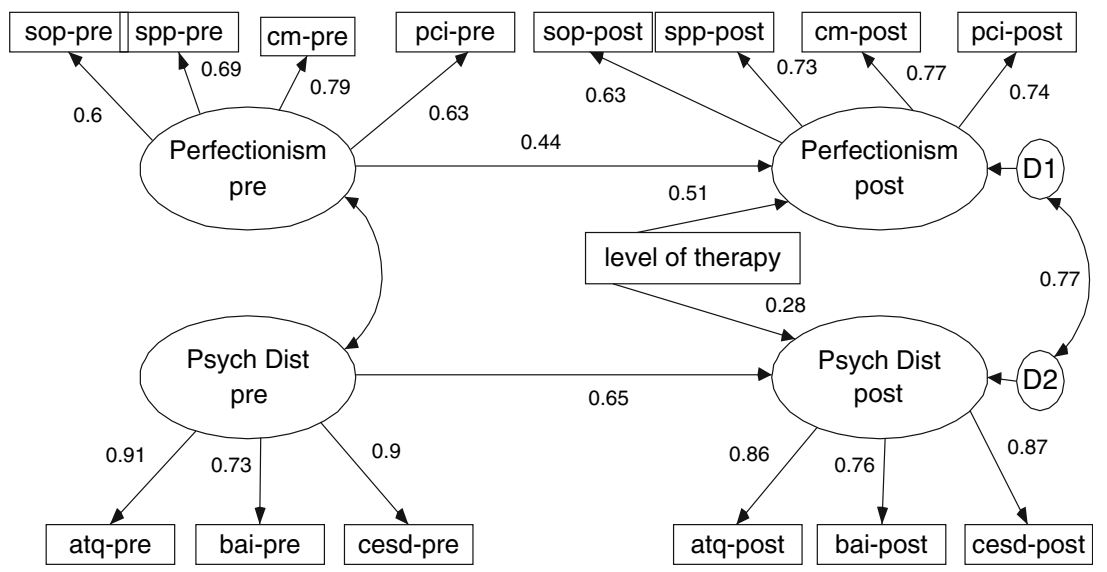

Fig. 1 The structural model used for investigating the relationship between the therapeutic outcomes for perfectionism and psychological distress

Bollen 1989) and root mean square error of approximation (RMSEA, Steiger and Lind 1980). Although the $\chi^{2}$ and RMSEA statistics are affected by sample size (the $\chi^{2}$ typically rejects models with larger sample sizes while the RMSEA often rejects models with smaller sample sizes, Gadelrab 2005; Jackson 2003), the CFI and IFI are more stable across sample sizes (Bollen 1989; Gadelrab 2005; Jackson 2003; Mulaik et al. 1989; Tanguma 2001).

\section{Results}

\section{Initial Screening}

The scores on each item of each scale were screened initially for the presence of missing data. It was found that less than $3 \%$ of the total data points were missing. Stochastic regression imputation was used to replace missing values on individual items by regressing the item with missing data on the remaining items in the scale and then adding a random residual error. Total scale (and subscale) scores were computed from the complete data set. For seven subjects missing posttest data, stochastic regression imputation was used to impute subscale scores, separately by condition. The total scale (and subscale) scores were screened for the presence of nonnormality and outliers, with no extreme nonnormality or outlying cases identified.

\section{Pretest Means Relative to Established Norms}

The pretest means displayed in Table 1 indicate that relative to existing norms, the participants in this sample as a whole were highly perfectionistic and highly distressed. The perfectionism measures are not surprising given that participants 
Table 1 Comparison of means (standard deviations) on each of the scales and subscales at pretest and posttest for each of level of therapeutic intervention

\begin{tabular}{|c|c|c|c|c|c|c|}
\hline \multirow[t]{2}{*}{ Scale } & \multicolumn{3}{|l|}{ Pretest } & \multicolumn{3}{|l|}{ Posttest } \\
\hline & GSMI/CBI & GSMI & NT & GSMI/CBI & GSMI & NT \\
\hline SOP & $\begin{array}{l}85.49 \\
(9.62)\end{array}$ & $\begin{array}{c}86.18 \\
(10.97)\end{array}$ & $\begin{array}{c}84.37 \\
(12.15)\end{array}$ & $\begin{array}{l}73.20^{* * *} \\
(10.98)\end{array}$ & $\begin{array}{c}80.31 \text { ** } \\
(14.08)\end{array}$ & $\begin{array}{c}85.17 \\
(14.53)\end{array}$ \\
\hline SPP & $\begin{array}{c}64.83 \\
(13.87)\end{array}$ & $\begin{array}{c}67.97 \\
(13.66)\end{array}$ & $\begin{array}{c}65.92 \\
(14.55)\end{array}$ & $\begin{array}{l}55.52^{* *} \\
(10.84)\end{array}$ & $\begin{array}{c}68.03 \\
(14.42)\end{array}$ & $\begin{array}{c}67.76 \\
(13.25)\end{array}$ \\
\hline $\mathrm{CM}$ & $\begin{array}{l}29.43 \\
(6.94)\end{array}$ & $\begin{array}{l}30.41 \\
(7.00)\end{array}$ & $\begin{array}{l}30.21 \\
(7.87)\end{array}$ & $\begin{array}{l}23.34 * * \\
(5.02)\end{array}$ & $\begin{array}{l}27.73^{* *} \\
(6.40)\end{array}$ & $\begin{array}{l}30.23 \\
(8.59)\end{array}$ \\
\hline PCI & $\begin{array}{c}66.14 \\
(15.55)\end{array}$ & $\begin{array}{c}68.83 \\
(10.53)\end{array}$ & $\begin{array}{c}69.75 \\
(12.50)\end{array}$ & $\begin{array}{l}50.24 * * \\
(15.72)\end{array}$ & $\begin{array}{l}60.15^{* *} \\
(17.10)\end{array}$ & $\begin{array}{c}70.36 \\
(12.35)\end{array}$ \\
\hline CESD & $\begin{array}{l}23.93 \\
(9.53)\end{array}$ & $\begin{array}{c}25.28 \\
(11.37)\end{array}$ & $\begin{array}{c}27.67 \\
(11.62)\end{array}$ & $\begin{array}{l}19.53 * \\
(8.08)\end{array}$ & $\begin{array}{c}24.65 \\
(12.70)\end{array}$ & $\begin{array}{l}27.00 \\
(9.62)\end{array}$ \\
\hline BAI & $\begin{array}{c}16.23 \\
(10.86)\end{array}$ & $\begin{array}{c}18.14 \\
(10.76)\end{array}$ & $\begin{array}{l}16.00 \\
(8.98)\end{array}$ & $\begin{array}{l}14.14 \\
(8.53)\end{array}$ & $\begin{array}{c}16.38 \\
(10.87)\end{array}$ & $\begin{array}{c}19.73 \\
(12.36)\end{array}$ \\
\hline ATQ & $\begin{array}{c}72.03 \\
(22.76)\end{array}$ & $\begin{array}{c}77.59 \\
(28.37)\end{array}$ & $\begin{array}{c}83.31 \\
(27.22)\end{array}$ & $\begin{array}{l}57.76^{* *} \\
(20.22)\end{array}$ & $\begin{array}{c}71.04 \\
(32.53)\end{array}$ & $\begin{array}{c}83.00 \\
(26.55)\end{array}$ \\
\hline
\end{tabular}

Note: GSMI/CBI = participants receiving cognitive behavioral and stress management therapeutic intervention; GSMI = participants receiving only the general stress management intervention; $\mathrm{NT}=$ participants receiving no therapeutic intervention; $*$, ** indicate significant change from pretest to posttest

$* p<.05, * * p<.01$

only qualified for this study if they had a PCI score at least one standard deviation above the established mean for this instrument. The participants in three groups had mean PCI scores of 66.14 or greater. Note that the mean PCI scores found recently in a mixed psychiatric patient sample and a second sample of patients recovery from alcohol use disorders were 46.79 and 53.59, respectively (see Flett et al., in press), so the overall level of perfectionism cognitions was quite high.

Further indication of the extreme levels of perfectionism in the sample as a whole comes from the high levels of self-oriented and socially prescribed perfectionism found at pretest. The self-oriented perfectionism means ranged from 84.37 to 86.18 for the participants in the three treatment conditions, while the mean socially prescribed perfectionism scores ranged from 64.83 to 67.97 . The respective means for self-oriented perfectionism and socially prescribed perfectionism for a normative sample of over 1100 university students were 68.0 for self-oriented perfectionism and 53.6 for socially prescribed perfectionism (see Hewitt and Flett 2004). The means for self-oriented and socially prescribed perfectionism in the current study exceed the means of 74.3 (self-oriented perfectionism) and 61.6 (socially prescribed perfectionism) reported for a normative sample of patients with diagnoses of unipolar depression (Hewitt and Flett 2004).

As for the levels of distress, the pretest means indicate substantially elevated levels of depression and anxiety. For instance, the reported CES-D means were 23.93 or higher and a score of 16 or higher is the cutoff for at least mild depression 
(Radloff 1977). As for the BAI means, mean pretest scores were 16.00 or higher across the three treatment conditions. These values far exceed the BAI mean of 6.6 found for a nationally representative community sample obtained in the United States (Gillis et al. 1995).

\section{Pretest to Posttest Differences on Measures}

Paired samples $t$-tests were used to compare pretest and posttest scores on each of the scales/subscales for each level of therapeutic intervention. These results are presented in Table 1. For GSMI/CBI participants, there were significant changes on all of the scales except the BAI. For GSMI participants, significant changes were noted on only three of the scale scores, those being the SOP, the CM, and the PCI. No significant changes were noted for NT participants.

\section{Structural Equation Modeling}

The structural model with standardized parameter estimates is displayed in Fig. 1. The fit of the model to the data was good, $\chi^{2}(\mathrm{df}=78)=105.71, p=.020$, $\mathrm{CFI}=.963, \mathrm{IFI}=.964, \mathrm{RMSEA}=.066[90 \% \mathrm{CI}=\{.027, .096)]$, with all standardized residuals (in absolute value) less than 3 .

As expected, posttest perfectionism was significantly predicted from pretest perfectionism, $z=4.27, p<.001$, and the posttest psychological distress factor was significantly predicted from pretest psychological distress factor, $z=6.27$, $p<.001$.The primary hypothesis of the study (i.e., level of therapeutic intervention would predict amount of change in perfectionism) was supported, $z=5.560$, $p<.001$. More specifically, those receiving more therapeutic intervention showed greater improvement than those receiving less therapeutic intervention. Level of therapeutic intervention also predicted amount of change in psychological distress, $z=2.774, p=.002$, where again those receiving more therapeutic intervention showed greater improvement than those receiving less therapeutic intervention. Further, as hypothesized, changes in perfectionism were significantly correlated with changes in psychological distress, $r=.773, p<.001$ (note that since pretest scores were factored out of posttest scores, the remaining variability in posttest scores can accurately be interpreted as changes, see Rausch et al. 2003), where those showing greater improvement in perfectionism also showed greater improvement in psychological distress.

\section{Discussion}

Perfectionism related concerns and the experience of psychological distress are important considerations at post-secondary institutions. Counseling and development centers across campuses regularly encounter students who struggle with perfectionism, with high levels of perfectionism in university students related to 
increased depression and anxiety levels, eating-disordered behavior, and comprised academic performance, to name only a few. The goal of this study was to investigate whether a short term, web-based, cognitive behavioral intervention could reduce levels of perfectionism and psychological distress in university students with elevated levels of perfectionism.

This study utilized a structural equation model in order to investigate all of the proposed relationships simultaneously, instead of in a 'piecemeal' fashion. The primary advantage of this approach is that it is assumes that behaviors (and for the purpose of this study, changes in behaviors) do not occur independently but instead are highly intertwined with related behaviors (and changes in related behaviors). The model proposed in this study allowed us to evaluate whether the level of therapeutic intervention provided to our participants was predictive of the amount of improvement in perfectionism and psychological distress, as well as whether the amount of improvement in perfectionism was related to the amount of improvement in psychological distress. Another important advantage of using structural equation modeling in this study was that the relationships investigated were between factors, or, in other words, we were able to investigate relationships among the unobserved latent constructs instead of the (measurement error prone) observed variables.

With regards to perfectionism, the results demonstrated that those receiving the stress management intervention showed significant improvement in self-oriented perfectionism and concern over mistakes. However, those receiving both the cognitive behavioral and stress management therapies (GSMI/CBI) showed significant improvement and greater overall improvement on all of the aspects of perfectionism investigated in this study (self-oriented, socially prescribed, concern over mistakes, and automatic perfectionistic thoughts). Further, the structural model demonstrated that the higher the level of therapeutic intervention, the greater the improvement on the perfectionism construct. Taken together, these results indicate that while the stress management intervention improved levels of perfectionism to some degree, the cognitive behavioral portion of the intervention contributed to improving perfectionism over and above the stress management intervention in isolation. This is important information for clinicians seeking the best form of treatment for extreme perfectionism, and supports the results of Kutlesa and Arthur (2008), who found that a group cognitive behavioral intervention significantly reduced perfectionism levels.

The results of this study also demonstrated that while the stress management intervention did not have a significant impact on depression, anxiety or automatic negative thoughts, participants receiving both the cognitive behavioral and stress management interventions showed significant improvement on depression and automatic negative thoughts. The structural model showed that the higher the level of therapeutic intervention, the greater the improvement on the latent measure of psychological distress. These results indicate that the cognitive behavioral portion of the intervention was important in promoting improvement in psychological distress.

An important direction for future research is to examine the effectiveness of a cognitive behavioral intervention when this type of intervention is the sole intervention and is not combined with a stress management component. While the 
current findings suggest that it is the cognitive behavioral aspect of the intervention that is most effective, there should also be some benefits that follow from a stress management approach. Perfectionists have been described as people who are highly responsive to stress and it has been suggested that by pursuing perfectionists standards, perfectionists generate extensive stress for themselves (for a review, see Hewitt and Flett 2002). Thus, the ability to manage stress should be a highly relevant theme for most perfectionists, and a combined treatment approach that incorporates this component is indicated.

When the relationship between changes in perfectionism and changes in psychological distress was investigated in the current study, it was found that the improvements on each are clearly not independent of one another. Specifically, changes in perfectionism were highly related to changes in psychological distress, with over half of the variability in changes in depression, anxiety and automatic negative thoughts being shared with changes in perfectionism $\left(r^{2}=.59\right)$. The fact that improvements in perfectionism were related to improvements in other forms of psychological distress provides further evidence to the fact that these attitudinal states are related and introduces the possibility that by effectively treating perfectionism we may also be reducing the likelihood that individuals may experience elevated levels of psychological distress.

We found clear evidence of the utility of a web-based intervention with combined cognitive behavioral and stress management features, but it is important to consider how our conclusions are qualified by some observations that may be inferred from the posttest results. First, although levels of depression and negative automatic thoughts were reduced, levels of anxiety were still elevated at posttest and well above normative values. This suggests the need to include additional treatment components that focus more directly on reducing symptoms of anxiety and anxietyrelated cognitions. Various themes related to anxiety were incorporated into the intervention component, but levels of anxiety were still elevated. A greater focus on anxiety is important given that anxiety and fear of failure have been described as central to an understanding of the development and maintenance of perfectionism (see Flett et al. 2002).

Second, although levels of perfectionism were substantially reduced at posttest for participants in the combined cognitive behavioral and stress management condition, overall levels of perfectionism cognitions and self-oriented perfectionism were still relatively high, relative to established norms, and some participants still had clear elevations on measures of perfectionism. Additional intervention would be beneficial given that the pattern of correlations found at posttest indicated that perfectionism was still associated with residual symptoms of depression and anxiety at posttest (see Table 2), despite the overall reductions in levels of perfectionism that were achieved as a result of the intervention.

\section{Limitations of the Current Study}

There are some limitations that are important to highlight in discussing the results of the present study. First, as with many psychological studies, the results are based on 
Table 2 Correlations among the variables used in the structural equation model

\begin{tabular}{|c|c|c|c|c|c|c|c|c|c|c|c|c|c|c|c|}
\hline & 1 & 2 & 3 & 4 & 5 & 6 & 7 & 8 & 9 & 10 & 11 & 12 & 13 & 14 & 15 \\
\hline 1. Intervention & 1 & & & & & & & & & & & & & & \\
\hline 2. SOP-pr & -.04 & 1 & & & & & & & & & & & & & \\
\hline 3. SOP-pt & .33 & .57 & 1 & & & & & & & & & & & & \\
\hline 4. SPP-pr & .04 & .34 & -.01 & 1 & & & & & & & & & & & \\
\hline 5. SPP-pt & .38 & .16 & .36 & .49 & 1 & & & & & & & & & & \\
\hline 6. CM-pr & .05 & .43 & .19 & .62 & .49 & 1 & & & & & & & & & \\
\hline 7. CM-pt & .37 & .22 & .48 & .34 & .65 & .56 & 1 & & & & & & & & \\
\hline 8. PCI-pr & .11 & .46 & .31 & .36 & .27 & .43 & .27 & 1 & & & & & & & \\
\hline 9. PCI-pt & .43 & .23 & .60 & -.04 & .48 & .18 & .53 & .43 & 1 & & & & & & \\
\hline 10. BAI-pr & .00 & .20 & .06 & .39 & .19 & .40 & .25 & .33 & .13 & 1 & & & & & \\
\hline 11. BAI-pt & .19 & .23 & .34 & .27 & .38 & .33 & .44 & .24 & .41 & .67 & 1 & & & & \\
\hline 12. CESD-pr & .14 & .06 & -.03 & .37 & .21 & .40 & .36 & .18 & .09 & .70 & .56 & 1 & & & \\
\hline 13. CESD-pt & .29 & .06 & .25 & .18 & .39 & .28 & .52 & .16 & .43 & .37 & .68 & .56 & 1 & & \\
\hline 14. ATQ-pr & .17 & .04 & -.07 & .45 & .28 & .44 & .32 & .27 & .08 & .66 & .47 & .82 & .47 & 1 & \\
\hline 15. ATQ-pt & .39 & .05 & .31 & .21 & .48 & .29 & .58 & .19 & .54 & .45 & .65 & .50 & .76 & .57 & 1 \\
\hline
\end{tabular}

Note: Intervention $=$ level of therapeutic intervention $(1=\mathrm{GSMI} / \mathrm{CBI}, 2=\mathrm{GSMI}, 3=\mathrm{NT})$; pr $=$ pretest; $\mathrm{pt}=$ posttest; $r>.216$ is statistically significant at $\alpha=.05$ and $r>.283$ is significant at $\alpha=.01$

self-report data and it is possible that the responses of the participants may not accurately represent their own personal reality. However, it should be made clear that in this study the individual perceptions of the participants (i.e., their responses to the items on the scales) are of utmost importance and any possible discrepancy between their perceptions and their actual behavior may not nullify the internal validity of the study.

Second, the intervention was set up in such a way that participants could vary considerably in the way they chose to make use of the intervention materials made available to them. For example, individuals were free to cover the material on whatever timeline they chose within the 10 -week window of web access. It is possible that when participants covered the material may have affected their responses on the measures at posttest. It will be important for future studies to be better able to assess and understand the way in which the participants are using the materials in order to identify those factors most closely related to overall levels of improvement.

Finally, the sample size in this study was relatively small and could have influenced the results of the structural equation modeling analyses. Small sample sizes can affect structural equation modeling analyses in a few ways, specifically increased presence of improper solutions (nonconvergence, negative error variances), reduced precision of parameter estimates, and low power for parameter estimates. However, it is our opinion that the sample size in this study did not impact on the goals or the conclusions of the study since no improper solutions were found (the model converged after only 11 iterations, and there were no negative variances), the model fit was acceptable even with the RMSEA (which tends to 
reject true models with small sample sizes), and all proposed relationships were statistically significant (so low power is not an issue). Therefore, the only limitation due to the sample size is the slightly reduced precision of the parameter estimates, which we hope will be rectified in future studies.

To summarize, this study has made a significant contribution to the understanding of the treatment of perfectionism and the relationship between the therapeutic outcomes for perfectionism and psychological distress. Our findings indicated clearly that a web-based intervention that combines stress management and cognitive behavioral techniques and themes is associated with significant reduction on core dimensions of perfectionism. It is hoped that future studies will investigate the generalizability of these findings with other methods of delivering the therapy, with differing lengths of treatment, and with different treatment populations.

\section{References}

Arbuckle, J. L. (2005). Amos 6.0 User's Guide. Chicago: SPSS Inc.

Barrow, J. C., \& Moore, C.A. (1983). Group intervention with perfectionistic thinking. The Personnel and Guidance Journal, 61, 612-615.

Beck, A. T., Epstein, N., Brown, G., \& Steer, R. A. (1988). An inventory for measuring clinical anxiety: Psychometric properties. Journal of Consulting and Clinical Psychology, 56, 893-897.

Bentler, P. M. (1989). EQS structural equations program manual. Los Angeles: BMDP Statistical Software.

Blatt, S. J., \& Zuroff, D. C. (2002). Perfectionism in the therapeutic process. In G. L. Flett \& P. L. Hewitt (Eds.), Perfectionism: Theory, research, and treatment (pp. 393-406). Washington, DC: American Psychological Association.

Bollen, K. A. (1989). Structural equations with latent variables. New York: Wiley.

Butler, S., Chalder, T., Ron, M., \& Wessely, S. (1991). Cognitive behavior therapy in chronic fatigue syndrome. Journal of Neurology, Neurosurgery, and Psychiatry, 54, 153-158.

Cox, B. J., \& Enns, M. W. (2003). Relative stability of dimensions of perfectionism in depression. Canadian Journal of Behavioural Science, 35, 124-132.

Deale, A., Chalder, T., Marks, I., \& Wessely, S. (1997). Cognitive behavior therapy for chronic fatigue syndrome: A randomized controlled trial. American Journal of Psychiatry, 154, 408-414.

Ellis, A. (2002). The role of irrational beliefs in perfectionism. In G. L. Flett \& P. L. Hewitt (Eds.), Perfectionism: Theory, research, and treatment (pp. 217-229). Washington, DC: American Psychological Association.

Ferguson, K. L., \& Rodway, M. R. (1994). Cognitive-behavioural treatment of perfectionism: Initial evaluation studies. Research on Social Work Practice, 4, 283-308.

Flett, G.L., \& Hewitt, P. L. (2006). Positive versus negative aspects of perfectionism in psychopathology: A comment on Slade and Owen's dual process model. Behavior Modification, 30, 472-495.

Flett, G. L., Hewitt, P. L., Blankstein, K., \& Gray, L. (1998). Psychological distress and the frequency of perfectionistic thinking. Journal of Personality and Social Psychology, 75, 1363-1381.

Flett, G. L., Hewitt, P. L., Blankstein, K., \& Koledin, S. (1991). Dimensions of perfectionism and irrational thinking. Journal of Rational-Emotive \& Cognitive-Behavior Therapy, 9, 185-201.

Flett, G. L., Hewitt, P. L., \& Dyck, D. G. (1989). Self-oriented perfectionism, neuroticism, and anxiety. Personality and Individual Differences, 10, 731-735.

Flett, G. L., Hewitt, P. L., Endler, N. S., \& Tassone, C. (1994). Perfectionism and components of state and trait anxiety. Current Psychology, 13, 326-350.

Flett, G. L., Hewitt, P. L., Garshowitz, M., \& Martin, T. R. (1997). Personality, negative social interactions, and depressive symptoms. Canadian Journal of Behavioural Science, 29, 28-37.

Flett, G. L., Hewitt, P. L., Oliver, J. M., \& Macdonald, S. (2002). Perfectionism in children and their parents: A developmental analysis. In G. L. Flett \& P. L. Hewitt (Eds.), Perfectionism: Theory, research, and treatment (pp. 89-132). Washington, DC: American Psychological Association Press. 
Flett, G. L., Hewitt, P. L., Whelan, T., \& Martin, T. R. (in press). The perfectionism cognitions inventory: Psychometric properties and associations with distress and deficits in cognitive self-management. Journal of Rational-Emotive and Cognitive-Behavior Therapy.

Friedberg, F. (1996). Chronic fatigue syndrome: A new clinical application. Professional Psychology, 27, 487-494.

Friedberg, F., \& Krupp, L. B. (1994). A comparison of cognitive behavioral treatment for chronic fatigue syndrome and primary depression. Clinical Infectious Diseases, 18, S105-S110.

Frost, R. O., Marten, P., Lahart, C., \& Rosenblate, R. (1990). The dimensions of perfectionism. Cognitive Therapy and Research, 14, 449-468.

Fukuda, K., Straus, S. E., Hickie, I., Sharpe, M. C., Dobbins, J. G., Komaroff, A., et al. (1994). Chronic fatigue syndrome: A comprehensive approach to its definition and study. Annals of Internal Medicine, 121, 953-959.

Gadelrab, H. F. (2005). The effect of model misspecification on overall goodness-of-fit indices for structural equation modeling. Doctoral dissertation, Wayne State University, Detroit, Michigan.

Gillis, M. M., Haaga, D. A. F., \& Ford, G. T. (1995). Normative values for the Beck anxiety inventory, fear questionnaire, Penn State worry questionnaire, and social phobia and anxiety inventory. Psychological Assessment, 7, 450-455.

Halgin, R. P., \& Leahy, P. M. (1989). Understanding and treating perfectionistic college students. Journal of Counselling and Development, 68, 222-225.

Hayward, L., \& Arthur, N. (1998). Perfectionism and post-secondary students. Canadian Journal of Counselling, 323, 187-199.

Hewitt, P. L., \& Dyck, D. G. (1986). Perfectionism, stress, and vulnerability to depression. Cognitive Therapy and Research, 10, 137-142.

Hewitt, P. L., \& Flett, G. L. (1990). Perfectionism and depression: A multidimensional analysis. Journal of Social Behavior and Personality, 5, 423-438.

Hewitt, P. L., \& Flett, G. L. (1991). Perfectionism in the self and social contexts: Conceptualization, assessment, and association with psychopathology. Journal of Personality and Social Psychology, 60, 456-470.

Hewitt, P. L., \& Flett, G. L. (2002). Perfectionism and stress in psychopathology. In G L. Flett \& P. L. Hewitt (Eds.), Perfectionism: Theory, research, and treatment (pp. 255-284). Washington: American Psychological Association Press.

Hewitt, P. L., \& Flett, G. L. (2004). The multidimensional perfectionism scale: Technical manual. Toronto: Multi-Health Systems, Inc.

Hewitt, P. L., Flett, G. L., Norton, G. R., \& Flynn, C. (1998). Dimensions of perfectionism and chronic symptoms of unipolar and bipolar depression. Canadian Journal of Behavioural Science, 30, 234242.

Hewitt, P. L., Mittelstaedt, W. M., \& Flett, G. L. (1990). Self-oriented perfectionism and generalized performance importance in depression. Individual Psychology, 46, 67-73.

Hollon, S. D., \& Kendall, P. C. (1987). Automatic thoughts questionnaire. In K. Corcoran \& J. Fischer (Eds.), Measures for clinical practice: A sourcebook (pp. 103-104). New York: Collier Macmillan.

Jackson, D. L. (2003). Revisiting sample size and number of parameter estimates: Some support for the N:q hypothesis. Structural equation modeling, 10, 128-141.

Joiner, T. E. Jr., \& Schmidt, N. B. (1995). Dimensions of perfectionism, life stress, and depressed and anxious symptoms: Prospective support for diathesis-stress but not specific vulnerability among male undergraduates. Journal of Social and Clinical Psychology, 14, 165-183.

Kutlesa, N. (2002). A group intervention with university students who experience difficulties with perfectionism (Doctoral dissertation, University of Calgary, 2002). Dissertation Abstracts International, 64(2-A), 398.

Kutlesa, N., \& Arthur, N. (2008). Overcoming negative aspects of perfectionism through group treatment. Journal of Rational-Emotive and Cognitive-Behavior Therapy.

Martin, T. R., Flett, G. L., Hewitt, P. L., Krames, L., \& Szanto, G. (1996). Personality correlates of depression and health symptoms: A test of self-regulation model. Journal of Research in Personality, 30, 264-277.

Mulaik, S. A., James, L. R., Van Alstine, J., Bennett, N., Lind, S., \& Stillwell, C. D. (1989). Evaluation of goodness of fit indices for structural equation models. Psychological Bulletin, 105, 430-445.

Rausch, J. R., Maxwell, S. E., \& Kelley, K. (2003). Analytic methods for questions pertaining to a randomized pretest, posttest, follow-up design. Journal of Clinical Child and Adolescent Psychology, 32, 467-486. 
Radloff, L. S. (1977). The CES-D scale: A self-report depression scale for research in the general population. Applied Psychological Measurement, 1, 385-401.

Radloff, L. S. (1987). Center for Epidemiologic Studies: Depressed mood scale. In K. Corcoran \& J. Fischer (Eds.), Measures for clinical practic: A sourcebook (pp. 118-119). New York: Collier Macmillan Inc.

Richards, P. S., Owen, L., \& Stein, S. (1993). A religiously oriented group counseling intervention for self-defeating perfectionism: A pilot study. Counseling and Values, 37, 96-104.

Ritterband, L. M., Gonder-Frederick, L. A., Cox, D. J., Clifton, A. D., West, R. W., \& Borowitz, S. M. (2003). Internet interventions: In review, in use, and into the future. Professional Psychology: Research and Practice, 34, 527-534.

Sharpe, M. C. (1996). Cognitive-behavioral therapy for patients with chronic fatigue syndrome: How? In M. A. Demitrack \& S. E. Abbey (Eds.), Chronic fatigue syndrome: An integrative approach to evaluation and treatment (pp. 212-239). New York: Guilford Press.

Steiger, J. H., \& Lind, J. M. (1980). Statistically based tests for the number of common factors. Paper presented at the annual meeting of the Psychometric Society, Iowa City, IA.

Tanguma, J. (2001). Effects of sample size on the distribution of selected fit indices: A graphical approach. Educational and Psychological Measurement, 61, 759-776. 
Reproduced with permission of the copyright owner. Further reproduction prohibited without permission. 